\title{
Resiliency Levels among Iraqi refugees in Jordan and Its Relation to Some Demographic Variables
}

\author{
Ashraf F. Alqudah ${ }^{1}$ \\ ${ }^{1}$ Department of Psychology, The University of Jordan, Amman, Jordan \\ Correspondence: Ashraf F. Alqudah, Department of Psychology, The University of Jordan, Amman, Jordan. Tel: \\ 962-785-422-478. E-mail: ashrafq2002@hotmail.com
}

Received: September 4, 2013

Accepted: September 17, $2013 \quad$ Online Published: November 5, 2013

doi:10.5539/ijps.v5n4p50

URL: http://dx.doi.org/10.5539/ijps.v5n4p50

\begin{abstract}
Refugees face enormous levels of stress post migration. These levels of stress put the refugees at risk for psychological and physical problems to add to their already existing burdens. Resiliency is known to reduce risk for stressors and increase ability to deal with burdens refugees face. 86 Iraqi refugees in Jordan participated in this study through 7 Non-Governmental Organizations (NGOs) working with Iraqi refugees in Jordan. Participants filled out the Connor-Davidson Resilience Scale (CD-RISC) and the Marlowe-Crowne Social Desirability Scale - Short Form (MC-SDS-SF). Results showed moderate resiliency levels amongst Iraqi refugees in Jordan. No differences between refugees' gender, age, and marital status were found. But differences between educational levels were found, indicating significantly higher resiliency scores for participants of higher educational levels. Results also showed that spiritual influence was the highest contributor to the refugees' resiliency. Implications of these results are discussed.
\end{abstract}

Keywords: refugees, resiliency, mental health, Jordan, Iraqi

\section{Introduction}

Millions of Iraqis have fled the violence spread in Iraq after the gulf war in 2003 to Jordan (Devi, 2007). About 2 million Iraqis fled to Jordan making it the first host country for Iraqi refugees. The exact number of them who stayed in Jordan is not clear. Numbers provided by Jordanian officials (180 000) and Non-Governmental Organizations (NGOs) (300 000) remain inconsistent (Chatelard, 2002).

Disasters resulting of any reason including armed conflicts can result in physical and psychological harm, both can take a toll on the psychological well-being of the affected population. Inherent issues of conflict, such as trauma, violence, life threats, uncertainty, separation from family and/or losing family members, poverty, depletion of financial reserve, and living in transient conditions can often make displaced populations experience an increased risk of mental health disorders.

One survey has looked at the prevalence of Iraqi refugees' need for mental health services in Jordan. The survey included 664 Iraqi refugees and found that $49 \%$ of them reported needing mental health services. $65 \%$ of the refugees who reported need for mental health, reported that the reason for needing mental health services was stress (Bader, Sinha, Leigh, Goyal, Andrews, Valeeva, Sirois, and Doocy, 2009).

A considerable body of literature has emerged on resilience and growth in response to trauma related to natural crises, physical illnesses, armed conflicts and bereavement (Schaefer and Moos, 1998). Schaefer and Moos (1998) showed that individuals who experienced life threatening situations frequently report a re-evaluation of their own lives and strengthened family relationships. Participants also indicated increased empathy for others and more frequent utilization of social resources such as family members, friends, and co-workers. Tedeschi and Calhoun (2004) also report that victims of trauma perceive that they have improved relationships, new possibilities, a greater appreciation for life, a greater sense of personal strength and spiritual development. However, limited research has examined resilience and coping strategies among persons who have been forced to flee their country of origin and become refugees. Resilient individuals show characteristics that seemed to be beneficial during and after exposure to stressors. They also show higher psychological functioning compared to individuals who did not show those characteristics (Iolli, Avicki, and Cepani, 2002). These characteristics 
include active response to military violence, creativity, and good and harmonious perceived parenting (Punamäki, Qouta and El-Sarraj, 2001).

Previous research indicates that post migration stressors were associated with symptoms of depression and anxiety (Schweitzer, Melville, Steel, and Lacharez, 2006). Also, Heptinstall, Sethna, and Taylor (2004) found that family financial difficulties, as well as having an insecure asylum status, were predictive of depression and PTSD. One survey study done by Fazel, Wheeler, and Danesh, (2005) found that refugees are approximately ten times more likely to have PTSD than age-matched native populations. Although the attention of most studies has been focusing on PTSD and major depression, increased rates of mental disorder relative to the domiciled population of generalized anxiety disorder, panic disorder, substance abuse and self-harm are also commonly reported (Bhugra, 2004). Co-occurrence of various combinations of these disorders are not uncommon (Craig, MacJajua, and Warfa, 2009).

There are multiple definitions of resilience. It has been defined as the ability to return to one's original functioning following exposure to a stressful or traumatic event, successful adaptation under challenging circumstances, and a trait of character or personality that enables positive adaptation to adversity and the timely attainment of psychological milestones (Masten and Coatsworth, 1998., Wilson, Zimmerman, and Whalen, 2000). Resilience also is affected by the personal qualities that make the individual able to thrive in the face of adversity (Connor and Davidson, 2003). Resiliency has also been defined as efforts to restore or maintain internal or external equilibrium under significant threat by means of human activities including thought and action (Smith and Carlson, 1997).

These definitions, to some extent, ignore the social context and the cross cultural dimensions of resilience the current research is focusing on (Gold, Engdahl, Eberly, Blake, Page, and Frueh, 2000., King, King, Foy, Keane, and Fairbank, 1999., and Werner-Wilson et al., 2000). The problem of defining resiliency is complicated by the way different cultures define violence (Hamby, 2000).

The multidimensionality of resilience has been demonstrated by research over the past 20 years. This multidimensionality varies across gender, age, context, cultural origins, and individual living environments (Garmezy, 1985., Garmezy and Rutter , 1985., Blackwell and Rutter , 1985., Seligman and Csikszentmihalyi , 2000., and Werner and Smith 1992). The variability of resilience dimensionality was proposed by the following resiliency model developed by Richardson and colleagues; Starting at the point of biopsychospiritual balance "homeostasis", the individual might adapt body, mind, and spirit to current life circumstances. Internal and external stressors are always present where the individuals' ability to cope with them is affected by the previous successful and unsuccessful efforts. In some situations, such efforts, or protective factors, are ineffective, resulting in disruption of the biopsychospiritual homeostasis. At the mean time, response to this disruption is a re-integrative process producing one of four outcomes: (1) the disruption forms an opportunity for growth and increased resilience, whereby efforts made towards dealing with the disruption leads to a new, higher level of homeostasis; (2) retreating to baseline homeostasis, which is an effort to just get past or beyond the disruption; (3) recovery with loss, establishing a lower level of homeostasis; or 4) a dysfunctional state, in which maladaptive strategies (e.g., self-destructive behaviors) are used to cope with stressors. Resilience may thus also be viewed as measure of successful stress-coping ability. (Richardson, Neiger, Jensen and Kumpfer, 1990., and Richardson, 2002).

In support of the contextual demotion of resilience, several researchers and clinicians have suggested that risk and resiliency cannot be understood apart from context (Grossman, Cook, Kepkep and Koenen, 1999., Harvey, 1996., and Hobfoll, Jackson, Hobfoll, Pierce and Young, 2002). Researchers and clinicians further warn that resilience is not a static trait, but rather an ongoing process in which new vulnerabilities and strengths arise during developmental, societal, and cultural transitions throughout one's life and during periods of acute stress and trauma (Laney, 1996). However, some contextual demotions of resiliency can enhance or reduce one's ability to be resilient. For example, communities can serve as sources of resilience by making needed resources available for individuals, or can serve as sources of additional turmoil and distress. Indeed, traumatic events can often disrupt support networks at the same time that they are most needed (Harvey, 1996., Koss and Harvey, 1991).

Cultural attitudes, beliefs, norms, and ideals play a crucial role in fostering and helping to maintain resilience after experiencing trauma. Self conceptualizing in relationship to the external world, and of the nature of traumatic experience, varies considerably across cultures. Traits and circumstances seen as promoting resilience in one culture may actually be considered as a liability in a different culture. For example, in individualistic cultures where individual achievements are highly valued, such as the United States and Western Europe, where 
personal autonomy and accomplishments, self-expression, and a strong sense of personal boundaries, are looked at as ideal. However, in collective cultures, such as the Middle Eastern, including Iraqi culture, interdependence with emphasis on the importance of maintaining and confirming bonds with family and community, are highly appreciated and practiced (Roland, 1996). In the collectively oriented cultures, an individual's reliance that exclude family values and obligations as guides for coping with interpersonal violence can be experienced as isolating to the individual and stressful to both the individual and his/her communities he or she identifies with. Hobfoll et al (2002) provide evidence to this notion when they found that participants high in communal-mastery experienced less increase in negative mood when faced with high stressors than participants who were low in communal-mastery. Good understanding of the uniqueness of trauma trajectories recovery in individuals from diverse cultures highlights the appreciation of resilience as a culturally shaped phenomenon. A resilient community will better face future hardships, may develop fewer symptoms of post-trauma, and enjoy a better quality of social support both within their own community and with neighboring ones (Doron, 2005).

This study is the first to study resiliency amongst Iraqi refugees in the Middle East. This study investigates the overall level of resiliency amongst Iraqi refugees in Jordan, and whether gender, age, educational levels, and marital statuses make any difference in resiliency amongst Iraqi refugees in Jordan. This study also investigates the factors that contribute to the Iraqi refugees' resiliency in Jordan.

\section{Method}

\subsection{Participants}

Eighty six Iraqi refugees in Jordan (62 females and 24 males) volunteered to participate in this study through 7 NGOs working with Iraqi refugees in Jordan. 18 years of age was the minimum age required to participate in this study. There were no restrictions on religious preference. Random epidemiological sampling was not feasible given the absence of a central register of refugees in Jordan and the geographical distribution of the target community. Participants agreed to participate in the study voluntarily. Participants were insured that their data will stay anonymous, confidential, and that it will not be used for any other purpose than scientific research. It was made clear to the participants that they can withdraw from participating in this study at any time and that there are no negative physical and/or psychological consequences are expected to take place due to participating in this study.

\subsection{Measures}

\subsubsection{The Connor-Davidson Resilience Scale (CD-RISC)}

The CD-RISC is a 25-item self-report assessment of psychological resilience. Total scores range from 0 to 100 , with higher scores reflecting greater resilience. The scale measure wide conceptions of resiliency and can be used as a clinical measure to assess treatment response. The scale measures five resiliency factors; (1) Personal competence, high standards, and tenacity, (2) Trust in one's instincts, tolerance of negative affect, and strengthening effects of stress, (3) Positive acceptance of change, and secure relationships, (4) Control, and (5)Spiritual influences. Items are assessed on a 5-point Likert scale that ranges from 'not true at all' (scored 0 ) to 'true nearly all the time' (scored 4). The CD-RISC has been shown to demonstrate excellent internal consistency and construct validity, particularly within the context of PTSD (Connor et al, 2003). Five experts in psychology and English language specialists have examined the Arabic translation and the back translation of the CD-RISC to assess for the translation validity of the measure. Internal consistency of the translated version of the CD-RISC was calculated and showed high internal consistency reliability (Cronbach alpha $=0.91$ ).

\subsubsection{Marlowe-Crowne Social Desirability Scale - Short Form (MC-SDS-SF)}

The MC-SDS-SF is a 10-item scale designed to measure social desirability though "Yes" "No" responses to the items. Five items of the scale are designed negatively and the other 5 items are designed positively. 5 experts in psychology and English language specialists have examined the Arabic translation and the back translation of the MC-SDS-SF to assess for the translation validity of the measure. Internal consistency of the translated version of the MC-SDS-SF was calculated and showed minimally adequate reliability (Cronbach alpha $=0.64)$.

\subsection{Statistical Analyses}

Descriptive statistics were conducted to summarize the demographic and background characteristics of the sample. Analyses of Variance were conducted to asses for group differences. Correlational analyses were conducted to test the correlational relationship between the independent variables (gender, age, educational level, and marital status) and the dependent variables (scores on the CD-RISC, and the total score of the MC-SDS-SF). 


\section{Results}

Descriptive statistics showed that $(27.6 \%)$ of the sample were males, and $(72.4 \%)$ were females. Participants' marital status was as follows: (24.6\%) single, $(64.9 \%)$ married, $(1.8 \%)$ divorced, $(7 \%)$ widowed, and $(1.8 \%)$ were engaged. Participants' educational levels were as follows: (16.1\%) below high school, (19.6\%) high school, (12.5) community college, (46.4) Bachelors degree, and (5.4) Masters degree. (50\%) of the participants were below age of (40), and (50\%) were above age of (40). Participants' average on the total score of the CD-RISC was (56) out (100), suggesting moderate resiliency. And (6.6) out of (10) on the MC-SDS-SF. Participants' averages on the CD-RISC are presented in Figure1 below.

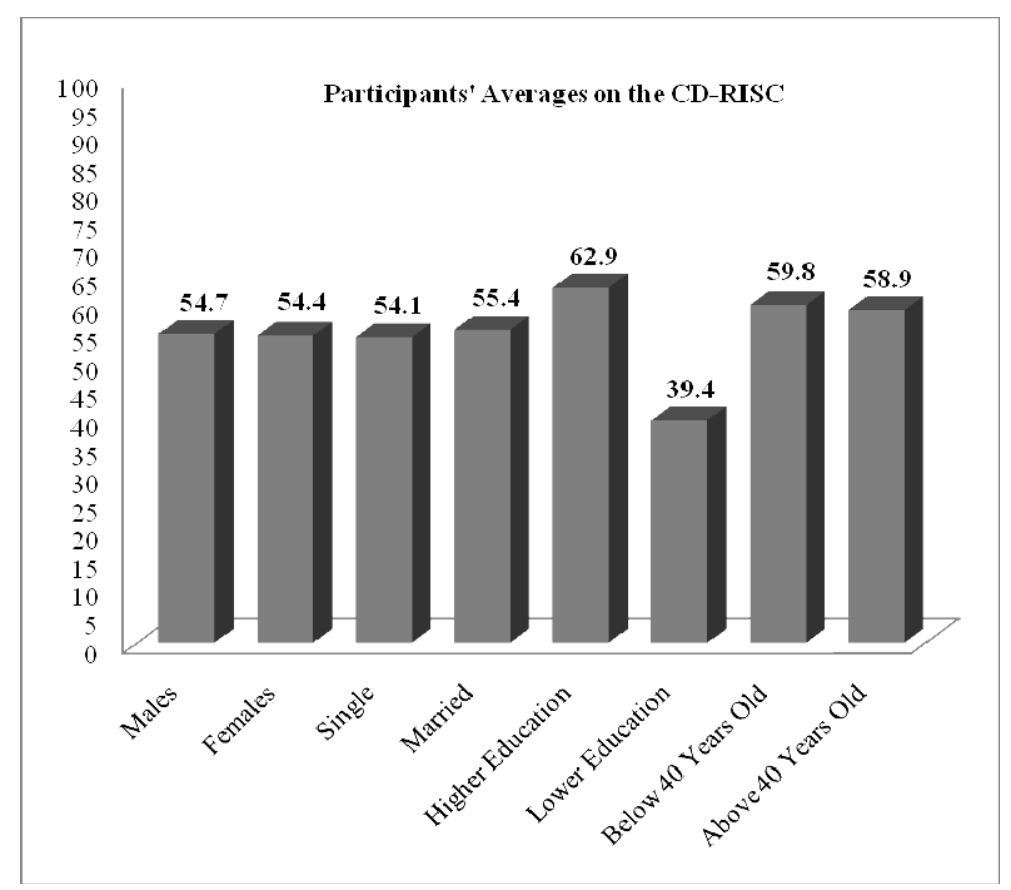

Figure 1. Participants' averages on the the CD-RISC

Participants' averages on the CD-RISC five factors showed that they have scored highest on the Spiritual Influences factor, and lowest on the factor of Trust in one's Instincts, Tolerance of Negative Affect, and Strengthening Effects of Stress. Participants' averages on the CD-RISC five factors are presented in figure 2 below. 


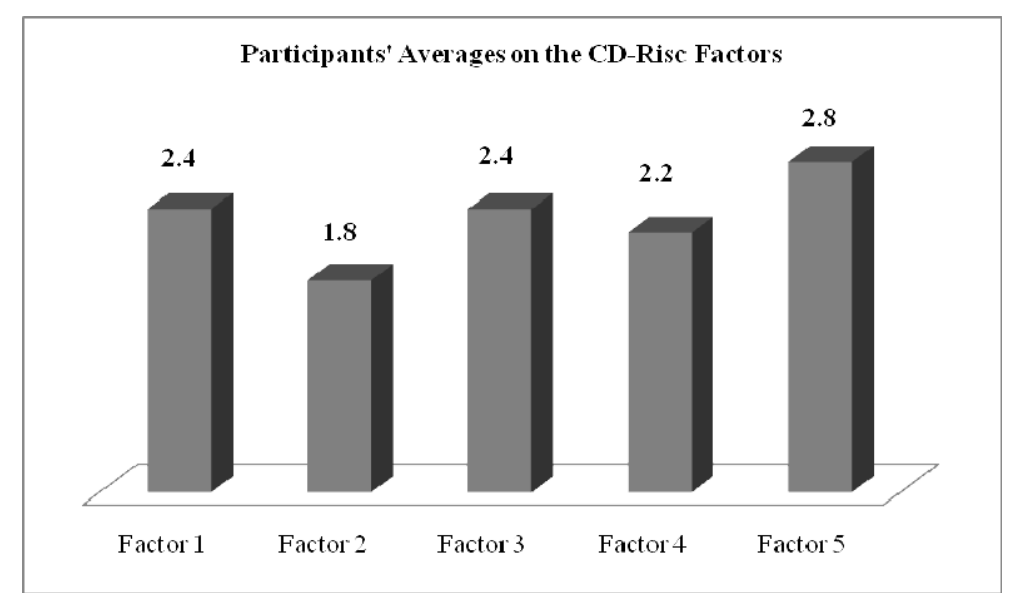

Figure 2. Participants' averages on the CD-RISC five factors

Factor 1. Personal competence, high standards, and tenacity

Factor 2. Trust in one's instincts, tolerance of negative affect, and strengthening effects of stress

Factor 3. Positive acceptance of change, and secure relationships

Factor 4. Control

Factor 5. Spiritual influences

Analyses of variance showed that there were no differences between men and women, between age groups (below 40 and above 40 years of age), and between martial statuses (married, single, widowed, divorced, and engaged). The only between groups difference was found between educational levels (below high school, high school, community college, bachelors degree, and masters degree), $\mathrm{F}(4,83)=3.82, \mathrm{p}<0.01$. Figure 3 below represents the overall educational levels and their total scores on the CD-RISC.

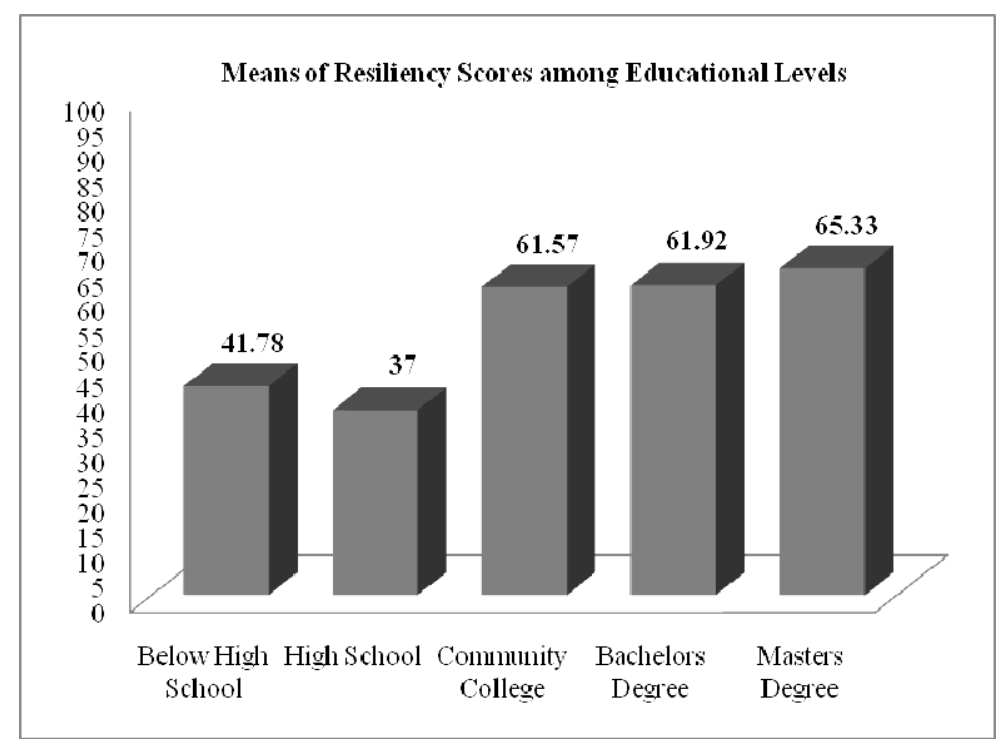

Figure 3. Educational levels and total scores on the CD-RISC

The differences between educational levels reflect two distinct levels of resiliency; a less than moderate resiliency, evidenced by the high school and lower educational levels' scores, and a significantly higher resiliency level, evidenced by the higher educational levels' scores. Table 1 below shows the means and standard deviations for the 5 educational levels. 
Table 1. Means and Standard deviations of participants' total CD-RISC scores based on their educational level

\begin{tabular}{lll}
\hline Educational level & M & SD \\
\hline Below High School & 41.78 & 23.95 \\
High School & 37.00 & 22.01 \\
Community College & 61.57 & 18.04 \\
Bachelors Degree & 61.92 & 19.52 \\
Masters Degree & 65.33 & 22.86 \\
\hline
\end{tabular}

Looking at the differences between educational levels on the five factors of the CD-RISC, significant differences emerge on the personal competence, high standards, and tenacity factor, $F(4,83)=4.08, p<0.01$, and on the positive acceptance of change, and secure relationships factor, $\mathrm{F}(4,83)=4.11, \mathrm{p}<0.01$, where participants of higher educational levels (community college, bachelors, and masters degrees) scored significantly higher on these two factors compared to lower educational levels (high school and below). Figure 4 below represents these results.

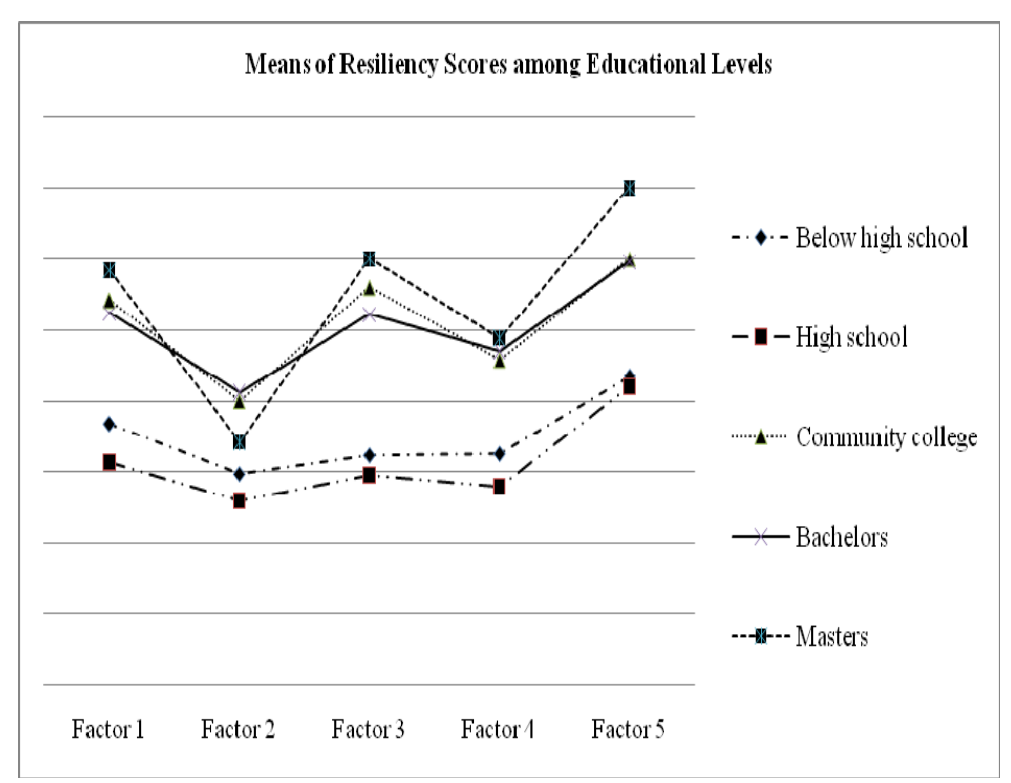

Figure 4. Means of Participants on the CD-RISC by their Educational Level

Factor 1. Personal competence, high standards, and tenacity

Factor 2. Trust in one's instincts, tolerance of negative affect, and strengthening effects of stress

Factor 3. Positive acceptance of change, and secure relationships

Factor 4. Control

Factor 5. Spiritual influences

Table 2 below presents the participants' educational levels averages and standard deviations on each of the five factors of the CD-RISC. 
Table 2. Participants' educational level averages on each of the five factors of the CD-RISC

\begin{tabular}{llllllllllll}
\hline & \multicolumn{3}{l}{ Factor 1 } & \multicolumn{3}{l}{ Factor 2 } & \multicolumn{3}{c}{ Factor 3 } & \multicolumn{3}{c}{ Factor 4 } & \multicolumn{2}{c}{ Factor 5 } \\
\cline { 2 - 10 } Educational Level & $M$ & $S D$ & $M$ & $S D$ & $M$ & $S D$ & $M$ & $S D$ & $M$ & $S D$ \\
\hline Below high school & 1.84 & .89 & 1.49 & 1.1 & 1.62 & .93 & 1.63 & 1.1 & 2.17 & 1.2 \\
High school & 1.57 & .98 & 1.3 & .86 & 1.48 & .93 & 1.4 & 1.0 & 2.1 & 1.1 \\
Community college & 2.71 & .84 & 2 & .77 & 2.8 & .83 & 2.29 & .95 & 3 & 1.0 \\
Bachelors & 2.62 & .81 & 2.06 & .91 & 2.61 & .95 & 2.35 & .95 & 2.98 & .99 \\
Masters & 2.92 & .95 & 1.71 & .90 & 3 & .69 & 2.44 & 1.1 & 3.5 & 1.1 \\
\hline
\end{tabular}

Factor 1. Personal competence, high standards, and tenacity

Factor 2. Trust in one's instincts, tolerance of negative affect, and strengthening effects of stress

Factor 3. Positive acceptance of change, and secure relationships

Factor 4. Control

Factor 5. Spiritual influences

Correlation analyses show that educational level is the only participants' characteristic that correlates with resiliency. It showed high correlation with the resiliency total score and with all resiliency factors except for the factor of Trust in one's Instincts, Tolerance of Negative Affect, and Strengthening Effects of Stress. Pearson correlation coefficients are presented in table 3 below.

Table 3. Pearson correlation coefficients between participants' age, gender, marital status, and educational level and the CD-RISC total score and the CD-RISC five factors

\begin{tabular}{lllllll}
\hline & Factor 1 & Factor 2 & Factor 3 & Factor 4 & Factor 5 & Total Score \\
\hline Age & .01 & -.03 & .03 & -.04 & -.13 & -.2 \\
Gender & -.02 & -.06 & .06 & .00 & .03 & -.01 \\
Marital Status & -.03 & .05 & -.08 & .04 & -.12 & -.04 \\
Educational Level & $.43^{* *}$ & .27 & $.47^{* *}$ & $.34^{*}$ & $.38^{* *}$ & $.43^{* *}$ \\
\hline
\end{tabular}

*. Correlation is significant at the 0.05 level

**. Correlation is significant at the 0.01 level

As for Social desirability, participants' scores on the MC-SDS-SF correlated significantly with their total scores on the CD-RISC. Participants' scores on the MC-SDS-SF also showed significant correlations with all resiliency factors except the factor of Trust in one's instincts, tolerance of negative affect, and strengthening effects of stress. Table 4 below presents Pearson correlation coefficients between participants' scores on the MC-SDS-SF, the CD-RISC, and the five factors of the CD-RISC.

Table 4. Pearson correlation coefficients between participants' scores on the MC-SDS-SF, the CD-RISC, and the five factors of the CD-RISC

\begin{tabular}{lllllll}
\hline & CD-RISC & Factor 1 & Factor 2 & Factor 3 & Factor 4 & Factor 5 \\
\hline MC-SDS-SF & $.30 * *$ & $.33 * *$ & .14 & $.28 *$ & $.85 * *$ & $.23 *$ \\
\hline
\end{tabular}

*. Correlation is significant at the 0.05 level

**. Correlation is significant at the 0.01 level 


\section{Discussion}

Resiliency is known to highly contribute to psychological and physical well being of individuals and communities (Doron, 2005). It is expected that the more resilient the refugee is, the more psychologically and physically he/she will be well. Current study results show that the overall resiliency level amongst Iraqi refugees in Jordan is moderate. This indicates that Iraqi refugees in Jordan are at risk for psychological and physical problems. This is consistent with Bader et al (2009) study, which found that approximately half of Iraqi refugees in Jordan are in need for psychological care. This is not surprising, however, since NGOs working with Iraqi refugees in Jordan report that most Iraqi refugees in Jordan find it hard to meet their basic needs. These needs include medical care, housing circumstances, specialized psychological care, labor opportunities etc. These unfulfilled needs can add to the toll on the refugees' psychological and physical health, especially those who are less resilient. Since the results of this study shows that they are moderately resilient, this means that approximately most of them will suffer as long as these needs are not met.

Current results indicate that there are no differences between genders, age groups, and marital statuses on resiliency levels. However, results show that there are differences between educational levels. The higher the educational level the higher the resiliency score. The lowest resiliency scores were reported by lower educational levels, while the highest scores were reported by the refugees who have higher educational levels. This may suggest that education contributes significantly to the resiliency of Iraqi refugees in Jordan. And that its contribution exceeds the contribution of gender, age, or marital status. Iraqi refugees in Jordan were not allowed by law to be admitted to public educational system from 2003 until 2007. These four years of not being able to attend education in Jordan may have adversely affected resiliency amongst Iraqi refugees. However, this resiliency obstacle does not exist anymore since Iraqi refugees are allowed to attend public educational system with full waver of tuition since 2007.

Correlation results show that education is the only refugee friable to correlate with the resiliency scores. It highly correlates with the resiliency total score and with three of the four factors of the CD-RISC (except for the factor of Trust in one's Instincts, Tolerance of Negative Affect, and Strengthening Effects of Stress.) This shows how significant education contributes to many factors that form the concept of resiliency. To increase resiliency amongst Iraqi refugees, these results suggest that it is important to provide educational opportunities to refugees in Jordan and anywhere and/or to provide Iraqi refugees with training programs aiming at enhancing their capacities and acquiring them knowledge and skills.

Although participants' scores on the MC-SDS-SF correlated significantly with their total scores on the CD-RISC, suggesting that social desirability may have had increased the Iraqi refugees' desire to present themselves as resilient, their overall resiliency stayed moderate.

Iraqi refugees did not score highly on all CD-RISC factors. The highest score was on the factor of Spiritual Influence. This suggests that spirituality is highly valued as a resiliency contributor amongst Iraqi refugees. This provides evidence to the variability of resilience dimensionality model proposed by Richardson and colleagues in 2002. It is, however, worth noticing that Iraqis have several spiritual preferences including Islam, Christianity, Sabi'a, and others. Comparison between spiritual preferences was out of the current study's scope. However, the current study recommends, based on the results, that hosting and fostering spiritual preferences of Iraqi refugees significantly enhances their resilience.

In summary, current study shows that there are no differences between genders, age groups, marital statuses in regard to resiliency among Iraqi refugees in Jordan. And, that higher education and spiritual influences are the highest two contributors to Iraqi refugees' resiliency. This study recommends that providing educational, training, and capacity building opportunities to Iraqi refugees and hosting and fostering their spiritual preferences enhance their overall resiliency.

\section{References}

Bader, F., Sinha, R., Leigh, J., Goyal, N., Andrews, A., Valeeva, N., Sirois, A., \& Doocy, S. (2009). Psychosocial Health in Displaced Iraqi Care-Seekers in Non-Governmental Organization Clinics in Amman, Jordan: An Unmet Need. Prehospital and Disaster Medicine, 24(4), 312-320.

Bhugra, D. (2004). Migration and mental health. Acta Psychiatr Scand, 109, $243 \mathrm{e} 58$. http://dx.doi.org/10.1046/j.0001-690X.2003.00246.x

Blackwell, \& Rutter. (1985). Resilience in the face of adversity: protective factors and resistance to psychiatric disorders. Br J Psych, 147, 598-611. http://dx.doi.org/10.1192/bjp.147.6.598 
Chatelard, G. (2002). Iraqi forced migrants in Jordan: Conditions, religious networks, and the smuggling process. Paper presented at the WIDER Conference on Poverty. International Migration and Asylum, 27-28.

Connor, K., \& Davidson. J. (2003). Development of a New Resilience Scale: The Conner-Davidson Resilience Scale (CD-RISC). Depression and Anxiety, 18, 76-82. http://dx.doi.org/10.1002/da.10113

Craig, T., MacJajua, P., \& Warfa, N. (2009). Mental health care needs of refugees. Psychiatry, 8(9). http://dx.doi.org/10.1016/j.mppsy.2009.06.007

Devi, S. (2007). Meeting the health needs of Iraqi refugees in Jordan. World Report, 370, December 1.

Doron, E. (2005). Working with Lebanese refugees in a community resilience model. Community Development Journal, 40(2), April, 182-191. http://dx.doi.org/10.1093/cdj/bsi026

Fazel, M., Wheeler, J., \& Danesh, J. (2005). Prevalence of serious mental disorder in 7000 refugees resettled in $\begin{array}{llllll}\text { western } & \text { countries: A systematic review. } & \text { Lancet, } & 365, & 1309 \mathrm{e} 14 .\end{array}$ http://dx.doi.org/10.1016/S0140-6736(05)61027-6

Garmezy, N. (1985). Stress resistant children: The search for protective factors. In Recent research in developmental psychopathology book (suppl number 4 to J Child Psychol Psych). Oxford: Pergamon Press.

Garmezy, N., \& Rutter, M. (1985). Acute stress reactions. In M. Rutter \& L. Hersob (Eds.), Child and adolescent psych: Modern approaches. Oxford.

Gold, P., Engdahl, B., Eberly, R., Blake, R., Page, W., \& Frueh, B. (2000). Trauma exposure, resilience, social support, and PTSD construct validity among former prisoners of war. Psychiatric Epidemiology, 35, 36-42. http://dx.doi.org/10.1007/s001270050006

Grossman, F., Cook, A., Kepkep, S., \& Koenen, K. (1999). With the phoenix rising: Lessons from ten resilient women who overcame the trauma of childhood rising: Lessons from ten resilient women who overcame the trauma of childhood sexual abuse. San Francisco: Jossey-Bass Publishers.

Hamby, S. (2000). The importance of community in a feminist analysis of domestic violence among America Indians. American Journal of Community Psychology, 28(5), 649-669. http://dx.doi.org/10.1023/A:1005145720371

Harvey, M. (1996). An ecological view of psychological trauma and trauma recovery. Journal of Traumatic Stress, 9(1), 3-23. http://dx.doi.org/10.1002/jts.2490090103

Heptinstall, E., Sethna, V., \& Taylor, E. (2004). PTSD and depression in refugee children: Associations with pre-migration trauma and post-migration stress. European Child and Adolescent Psychiatry, 13, 373-380. http://dx.doi.org/10.1007/s00787-004-0422-y

Hobfoll, S., Jackson, A., Hobfoll, I., Pierce, C., \& Young, S. (2002). The impact of b communal-mastery versus self-mastery on emotional outcomes during stressful conditions: A prospective study of Native American women. American Journal of Community Psychology, 30(6), 853-871. http://dx.doi.org/10.1023/A:1020209220214

King, D., King, L., Foy, D., Keane, T., \& Fairbank, J. (1999). Posttraumatic stress disorder in a national sample of female and male Vietnam veterans: Risk factors, war zone stressors, and resilience-recovery variables. Journal of Abnormal Psychology, 108(1), 164-170. http://dx.doi.org/10.1037/0021-843X.108.1.164

Koss, M., \& Harvey, M. (1991). The rape victim: Clinical and community interventions. In Thousand Oaks. CA: Sage Publications.

Laney, M. (1996). Multiple personality disorder: Resilience and creativity in the preservation of the self. Psychoanalysis and Psychotherapy, 13(1), 35-49.

Iolli, L., Avicki, V., \& Cepani. A. (2002). Resilience in the Face of Catastrophe: Optimism, Personality, and Coping in the Kosovo Crisis. Journal of Applied Social Psychology, 32(8), 1604-1627. http://dx.doi.org/10.1111/j.1559-1816.2002.tb02765.x

Masten, A., \& Coatsworth, J. (1998). The development of competence in favorable and unfavorable environments: Lessons from research on successful children. American Psychologist, 53(2), 205-220. http://dx.doi.org/10.1037/0003-066X.53.2.205

Punamäki, R., Qouta, S., \& El-Sarraj, E. (2001). Resiliency factors predicting psychological adjustment after political violence among Palestinian Children. International Journal of Behavioral Development, 25(3), 256-267. http://dx.doi.org/10.1080/01650250042000294 
Richardson, G. (2002). The metatheory of resilience and resiliency. J Clin Psychol, 58, 307-321. http://dx.doi.org/10.1002/jclp.10020

Richardson, G., Neiger, B., Jensen, S., \& Kumpfer, K. (1990). The resiliency model. Health Education, 21, 33-39.

Roland, A. (1996). Cultural pluralism and psychoanalysis: The Asian and North American experience. New York: Routledge.

Schaefer, J., \& Moos, R. (1998). The context for posttraumatic growth: Life crises, individual and social resources, and coping. In R. Tedeschi, C. Park, \& L. Calhoun (Eds.), Posttraumatic Growth: Positive Changes in the Aftermath of Crises (pp. 99-125). London: Laurence Erblaum Associates.

Schweitzer, R., Melville, F., Steel, Z., \& Lacharez, P. (2006). Trauma, post-migration living difficulties, and social support as predictors of psychological adjustment in resettled Sudanese refugees. Australian and New Zealand Journal of Psychiatry, 40, 179-187. http://dx.doi.org/10.1080/j.1440-1614.2006.01766.x

Seligman, \& Csikszentmihalyi. (2000). Positive psychology. Am Psychologist, 55, 5-14. http://dx.doi.org/10.1037/0003-066X.55.1.5

Smith, C., \& Carlson, B. (1997). Stress, coping, and resilience. Social Service Review, 71, 231-257. http://dx.doi.org/10.1086/604249

Tedeschi, R., \& Calhoun, L. (2004). Posttraumatic growth: A new perspective on psychotraumatology. Psychiatric Times, 21, 58-60.

Werner, E., \& Smith, R. (1992). Overcoming odds: High risk children from birth to adulthood. In Ithaca. NY: Cornell University Press.

Wilson, R., Zimmerman, T., \& Whalen, D. (2000). Resilient response to battering. Contemporary Family Therapy, 22(2), 161-188. http://dx.doi.org/10.1023/A:1007777702757

\section{Copyrights}

Copyright for this article is retained by the author(s), with first publication rights granted to the journal.

This is an open-access article distributed under the terms and conditions of the Creative Commons Attribution license (http://creativecommons.org/licenses/by/3.0/). 